\title{
EFECTOS DE DIFERENTES TIPOS DE ENTRENAMIENTO EN EL APRENDIZAJE DE UNA DISCRIMINACIÓN CONDICIONAL
}

\author{
María Elena Rodríguez Pérez ${ }^{1}$, Luis Hernando Silva Castillo ${ }^{1}$, Luis René Bautista Castro ${ }^{2}$ y Telmo Eduardo Peña Correal ${ }^{3}$ \\ ${ }^{1}$ Universidad de Guadalajara - México, ${ }^{2}$ Universidad Veracruzana - México, ${ }^{3}$ Universidad del Rosario - Colombia
}

Recibido, octubre 11/2014

Concepto de evaluación, noviembre 23/2014

Aceptado, diciembre 30/2014
Referencia: Rodríguez Pérez, M. E., Silva Castillo, L.H., Bautista Castro, L.R. \& Peña Correal, T.E. (2015). Efectos de diferentes tipos de entrenamiento en el aprendizaje de una discriminación condicional. Acta Colombiana de Psicología, 18(1), 55-67. DOI: 10.14718/ACP.2015.18.1.6

\section{Resumen}

\begin{abstract}
Se diseñó un experimento para analizar el papel funcional de la retroalimentación, las instrucciones y la observación de un modelo aprendiz empleando una tarea de igualación a la muestra de segundo orden. Treinta y nueve estudiantes se asignaron aleatoriamente a uno de los tres grupos experimentales que difirieron en el entrenamiento: exposición directa a la tarea, observación de un modelo aprendiz (confederado), o uso de instrucciones precisas sobre los criterios de igualación. A algunos participantes se les ofreció un incentivo económico por su participación. Se encontró que las instrucciones precisas promovieron un aprendizaje más acelerado en comparación con los otros tipos de entrenamiento. El empleo del incentivo económico tuvo un efecto diferencial según el tipo de entrenamiento; hizo que los participantes tuvieran un mejor desempeño en la condición de observación del modelo aprendiz, pero no influyó en los otros tipos de entrenamiento. Estos resultados sugieren que el incentivo puede operar como un factor motivacional o disposicional, dado que afecta la probabilidad del establecimiento del control abstracto del estímulo.

Palabras clave: discriminación condicional, modelado, retroalimentación, instrucciones, conducta mediada lingüísticamente
\end{abstract}

\section{EFFECTS OF DIFFERENT TYPES OF TRAINING IN LEARNING A CONDITIONAL DISCRIMINATION}

\begin{abstract}
An experiment was designed to analyze the functional role of feedback, instructions and observation of a learning model using a second-order matching-to-sample task. Thirty nine students were randomly assigned to one of the three experimental groups which differed among them in the type of training used: direct exposure to the task, observation of a learning model (confederate) or use of precise instructions about the matching criteria. Some participants were offered an economic incentive for participation. It was found that precise instructions promoted a faster acquisition than the other types of training. The use of economic incentives had a differential effect depending on the type of training. It promoted a high performance on the modeling condition but it did not have any effect on the other two types of training. These results suggest that incentives can operate like a motivational or dispositional factor since they affect the establishment of abstract stimulus control.

Key words: conditional discrimination, modeling, feedback, instructions, linguistic mediated behavior
\end{abstract}

* Centro de Estudios e Investigaciones en Comportamiento Universidad de Guadalajara. Francisco de Quevedo \# 180. Col. Arcos Vallarta. Guadalajara, Jalisco, México. C.P. 44130. Tel. 52 - 33 - 38180730 Ext.33307.rpm08428@cucba.udg.mx, hernando1427@gmail.com, renebautista81@hotmail.com, telmo.pena@urosario.edu.co. La presente investigación formó parte del trabajo interinstitucional entre la Universidad de Guadalajara y la Universidad Nacional de Colombia que se llevó a cabo bajo el apoyo a la Red Temática de Colaboración "Conducta Humana Compleja" reconocida por el Programa de Mejoramiento del Profesorado (PROMEP) en México. 


\title{
EFEITOS DE DIFERENTES TIPOS DE TREINAMENTO NA APRENDIZAGEM DE UMA DISCRIMINAÇÁO CONDICIONAL
}

\author{
Resumo
}

\begin{abstract}
Criou-se um experimento para analisar o papel funcional da retroalimentação, as instruções e a observação de um modelo aprendiz empregando uma tarefa de igualação à mostra de segunda ordem. Trinta e nove estudantes foram distribuídos aleatoriamente em três grupos experimentais que diferiram no treinamento: exposição direta à tarefa, observação de um modelo aprendiz (confederado), o uso de instruções exatas sobre os critérios de igualação. A alguns participantes foi oferecido um incentivo econômico pela sua participação. Encontrou-se que as instruções exatas promoveram uma aprendizagem mais acelerada em comparação com os outros tipos de treinamento. O emprego do incentivo econômico teve um efeito diferencial segundo o tipo de treinamento; fez com que os participantes tivessem um melhor desempenho na condição de observação do modelo aprendiz, mas não influiu nos outros tipos de treinamento. Estes resultados sugerem que o incentivo pode operar como um fator motivacional ou disposicional, dado que afeta a probabilidade do estabelecimento do controle abstrato do estímulo. Palavras chave: discriminação condicional, modelado, retroalimentação, instruções, conduta mediada linguisticamente
\end{abstract}

\section{INTRODUCCIÓN}

El análisis experimental del comportamiento verbal ha enfocado su estudio a partir de la distinción Skinneriana entre la conducta moldeada por contingencias y la conducta gobernada por reglas (Skinner, 1969/79). En ese contexto se ha investigado sistemáticamente sobre el fenómeno conocido como "control instruccional", en que estímulos previos a la conducta (denominados "instrucciones") cumplen la función de orientar y guiar el comportamiento subsecuente. Estas descripciones o instrucciones tienen diversos efectos sobre la conducta. Según Ribes (1990), el que más se destaca es la simplificación del proceso de aprendizaje del individuo, impidiendo que responda incorrectamente. Es decir, que la ejecución de los participantes a los que se les proporcionan instrucciones tiende a ser más efectiva que la de aquellos a los cuales no se les suministra esa guía, porque gracias a lo explicitado en la instrucción se reduce la variabilidad conductual que puede conducir a errores. En un sentido semejante, Skinner (1969/79) plantea que los individuos que son expuestos a instrucciones aprenden más rápidamente y sin necesidad de cometer errores, reduciendo el rango posible de respuestas en la situación.

Las tareas de igualación a la muestra se han empleado como ejemplar metodológico en la investigación empírica sobre el papel de variables lingüísticas en la explicación del comportamiento humano (Ribes, Ibañéz \& Hernández-Pozo; 1986, Ribes \& López, 1985; Ribes \& Zaragoza, 2009). En este procedimiento, se presentan cuando menos tres estímulos: uno denominado "muestra" y dos estímulos "comparativos". Se retroalimenta positivamente la respuesta al estímulo comparativo que guarde una relación con el estímulo muestra con base en un criterio predeterminado tal como la identidad, diferencia o semejanza (Ribes, 1990; Goldiamond, 1966;
Islas \& Flores, 2007). No obstante, autores como Goldiamond (1966) sugieren una distinción entre propiedades dimensionales del estímulo (como modalidades físicas a las que se atiende para igualar) y propiedades instruccionales (en el sentido en que "indican" la relación relevante entre propiedades dimensionales), de modo que, tanto estímulos con morfología verbal como aquellos que no la tienen podrían ser funcionalmente semejantes. Es decir, pueden tener las mismas funciones instruccionales descritas previamente en tanto que permitan "responder a propiedades variantes de los estímulos presentes en términos de una propiedad constante que es relacional: la correspondencia de propiedades entre el estímulo de muestra y uno de los estímulos de comparación" (Ribes, Torres, Barrera \& Ramírez, 1995: 116). Este proceso de discriminación relacional es lo que Goldiamond (1966) denomina control abstracto de estímulo.

En este sentido, las preparaciones usuales con humanos bajo este ejemplar metodológico incluyen un segmento instruccional ampliado o redundante, en tanto que incluyen no sólo estímulos que indican la relación, sino además, estímulos verbales que explicitan esta función. No obstante, atendiendo a la lógica de la propuesta Skinneriana, se ha atribuido principalmente el rol instruccional al estímulo verbal antecedente. En consecuencia, se han comparado los efectos de diferentes tipos de instrucciones y formas de retroalimentación de la ejecución instrumental en el aprendizaje de la discriminación condicional, así como sus efectos diferenciales en pruebas de transferencia. De hecho, hasta el momento, la evidencia recabada parece privilegiar estas comparaciones (González \& Ortiz, 2014; Guzmán \& Serrano, 2013; Islas \& Flores, 2007; Ortiz \& González, 2010; Ortiz, González, Rosas \& Alcaraz; 2006, Pérez y Pereira, 1986; Ribes \& Martínez, 1990; Villanueva, Mateos \& Flores, 2008). 
Así mismo, y asumiendo que la función instruccional no se deriva necesariamente de la morfología de los estímulos antecedentes a una respuesta de igualación, autores como Ribes (1990) sugieren que una tercera fuente de información que promueve el aprendizaje (cuando menos en contextos de desarrollo de competencias) es la demostración o entrenamiento por observación. Por tanto, desde una perspectiva que privilegia nexos funcionales sobre distinciones formales se abre la posibilidad de indagar empíricamente sobre dichos nexos o equivalencias, de modo que se han comparado directamente estas tres condiciones: entrenamientos de tipo instrumental e instruccional con un entrenamiento de tipo observacional (Ribes \& Castillo 1998; Ribes, Moreno \& Martínez, 1998; Ribes, Rodríguez \& Fuentes, 2003; Vega \& Peña 2008). Otras fuentes de evidencia también sugieren la relevancia de la comparación dado que muestran que es posible el aprendizaje de relaciones entre estímulos sin necesidad de la intervención de la respuesta directa de igualación (Tonneau, Arreola \& Martínez 2006; Tonneau \& González, 2004).

No obstante, este tipo de investigaciones no suelen contemplar directamente el papel de diversos tipos de modelos en términos de posibles diferencias en su ejecución. De alguna manera, los modelos enseñan la forma correcta de llevar a cabo la tarea y esto no necesariamente reproduce las condiciones en las que tiene lugar el aprendizaje mediante la respuesta de igualación, precedida o no de instrucciones, porque dichas condiciones implican instancias positivas y negativas de la respuesta. Al respecto, Ribes (2000) sugiere que tal tipo de experiencia es la que distingue la conducta de seguir instrucciones de la conducta de construir reglas.

Un trabajo que resulta de interés debido a que evalúa directamente el papel de diversos tipos de modelos, es el estudio de Vega \& Peña (2008) en el que se emplearon instrucciones, retroalimentación y modelos (confederados y no confederados) para evaluar su equivalencia funcional en un diseño de cinco grupos. En el grupo 1, el aprendizaje se promovió a través de la exposición directa a la tarea. Los participantes recibieron instrucciones mínimas pero se retroalimentaron sus respuestas de igualación con las palabras "acierto" o "error" según si la respuesta era correcta o incorrecta. El grupo 2 también aprendió a través de la retroalimentación de su ejecución, pero fue observado, mientras aprendía, por participantes de un tercer grupo. Éstos pasaron directamente a la condición de prueba después de haber observado al modelo aprendiz. Los participantes de un cuarto grupo observaron a un confederado mientras resolvía la tarea de igualación con $100 \%$ de aciertos. Por tal motivo, a esta condición se le denominó observación de un modelo experto. Por último, a los participantes del grupo 5 se les dieron instrucciones explícitas sobre las modalidades pertinentes que debían tomar en cuenta en los ensayos de semejanza o diferencia. Los resultados mostraron que los participantes del grupo con instrucciones precisas tuvieron mejor desempeño (en la adquisición y transferencia) que los grupos entrenados mediante exposición directa a la tarea. Además, quienes observaron al modelo experto lograron desempeños (en la condición de prueba) similares a los entrenados por exposición directa a la tarea. Un hallazgo interesante fue el hecho de que prácticamente ninguno de los participantes expuestos a la condición de observación de modelos aprendices aprendió la discriminación condicional. Esto contradice el supuesto de la necesidad de la experiencia "mixta" (exposición a instancias positivas y negativas) para el aprendizaje de relaciones condicionales.

Debido a que en investigaciones anteriores no se han controlado variables relacionadas con la ejecución de los modelos aprendices (patrones de respuestas y latencias), no se han podido realizar juicios confiables sobre el papel funcional de la observación de un modelo aprendiz y no se ha logrado comparar directamente con otros tipos de entrenamiento. Adicionalmente, la comparación entre entrenamientos se ha realizado en tareas de discriminación condicional de primer orden. Por estas razones, la presente investigación se planteó con el objetivo de comparar directamente los efectos de tres condiciones en el aprendizaje de una discriminación condicional: por exposición directa a la tarea, por modelado, o por instrucciones precisas. A pesar de que el modelo aprendiz en Vega \& Peña (2008) no promovió el aprendizaje, en esta investigación se utilizó un modelo aprendiz con el fin de privilegiar la equivalencia con las otras dos condiciones, al menos en términos de su exposición a instancias de retroalimentación tanto positiva como negativa. A diferencia de Vega \& Peña, se garantizó que la ejecución modelada fuera la de un aprendiz exitoso, es decir, de un aprendiz que identifica progresivamente el criterio de igualación correcta.

Por otra parte, dado que hay un especial interés en evaluar la complejidad del aprendizaje promovido por cada tipo de entrenamiento, entendida en términos de su extensión a situaciones diferentes a las del entrenamiento, en la presente investigación se emplearon pruebas de transferencia usuales en esta tradición (Varela \& Quintana, 1995). Sin embargo, dado que estas pruebas han mostrado no ser suficientemente sensibles a diferencias encontradas en el entrenamiento, se incluyó una prueba de transferencia adicional que requirió la construcción de relaciones de igualación a partir de la aplicación explícita de un criterio de resolución identificado verbalmente como producto de la experiencia durante la tarea. Bautista (2010) empleó una prueba semejante con el objetivo de establecer justamente la extensión del control abstracto del estímulo en una tarea de igualación a la muestra 
encontrando que, si bien no fue una condición suficiente, únicamente los participantes que fueron capaces de formular criterios verbales abstractos de ejecución lograron resolver adecuadamente la prueba de construcción.

\section{MÉTODO}

\section{Participantes}

Para esta investigación se invitó a participantes de dos poblaciones distintas: estudiantes de ingeniería de la Universidad de Guadalajara (México) y estudiantes de psicología de la Universidad Nacional de Colombia. Por tanto, el estudio se llevó a cabo en dos escenarios experimentales. En Guadalajara, los participantes trabajaron en el laboratorio del Centro de Estudios e Investigaciones en Comportamiento, el cual contó con cubículos aislados del ruido de 2 × 2 metros, con buena iluminación, ventilación y temperatura regulada. Cada cubículo estuvo equipado con una mesa, sillas y una computadora personal de escritorio Pentium Dual-Core, con teclado y ratón en donde se presentó la tarea experimental de manera individual. En Bogotá, los participantes trabajaron de manera individual en el Laboratorio de Conducta Simbólica con condiciones similares, lo cual aseguró la equivalencia de los escenarios experimentales. Por tanto, los datos aquí reportados corres- ponden a 20 estudiantes de la Universidad de Guadalajara cuyo rango de edad estaba entre 19 y 31 años (media de 22.2, desviación estándar de 3.3 años) y 19 estudiantes de la Universidad Nacional de Colombia cuyo rango se encontraba entre 17 y 34 años (media de 21.3 y desviación estándar de 4.3 años).

\section{Tarea Experimental}

Se utilizó una tarea de igualación a la muestra de segundo orden que se programó en el software Macromedia Authorware 7.0. En la aplicación se presentaron en la parte superior de la pantalla dos estímulos (de segundo orden o selectores) que mostraron la relación que sería retroalimentada positivamente en cada ensayo. Además, apareció un estímulo ubicado en el centro de la pantalla (estímulo muestra) y tres estímulos ubicados en la parte inferior (estímulos de comparación). El participante tenía que seleccionar uno de los estímulos de comparación de acuerdo con la relación prescrita por los estímulos de segundo orden al presionar el botón izquierdo del ratón sobre la figura elegida.

Los estímulos utilizados y los criterios de igualación se encuentran en la figura 1. Los criterios de igualación entrenados y evaluados en el experimento fueron la identidad y

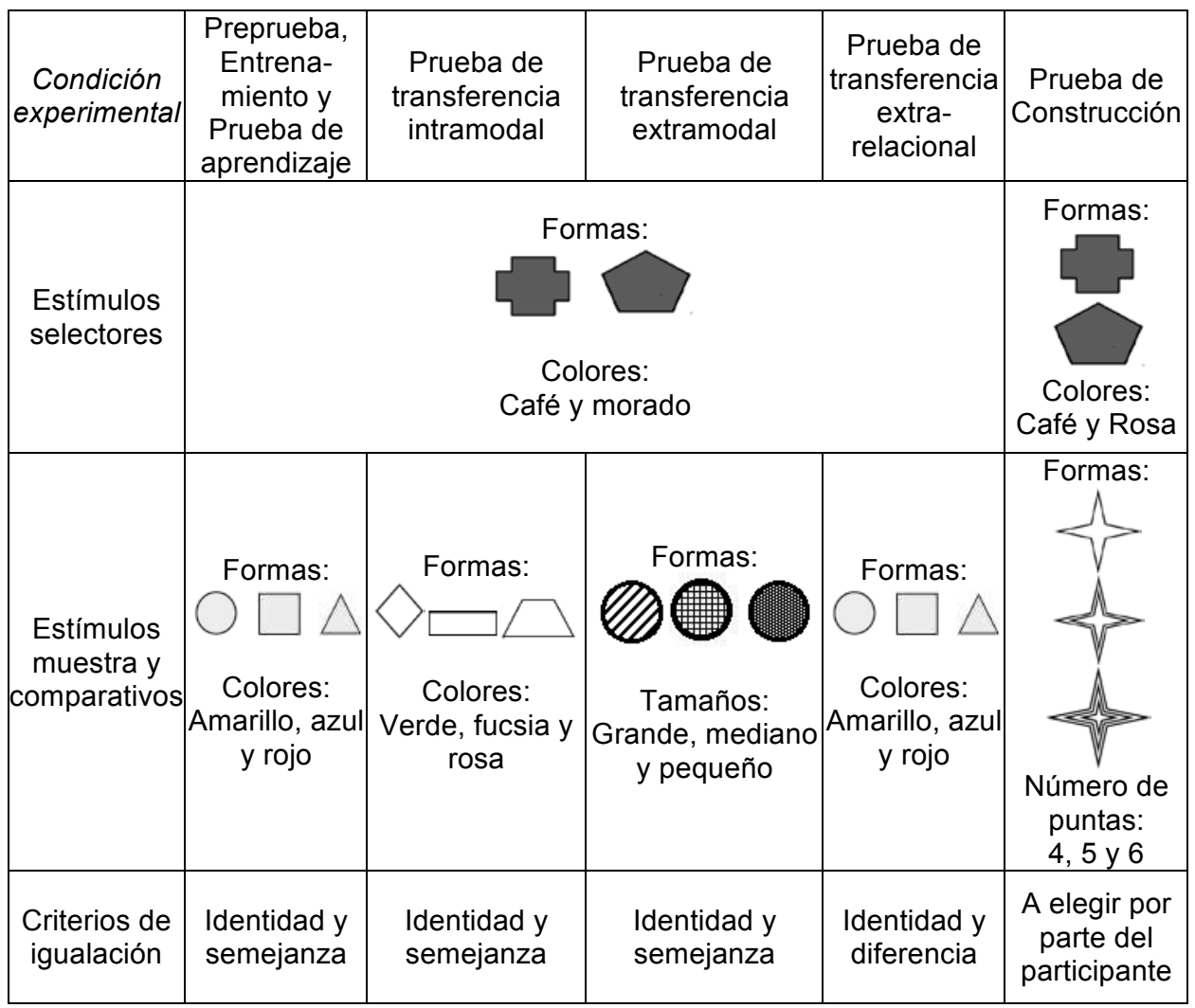

Figura 1. Estímulos y criterios utilizados en la tarea de igualación durante las distintas condiciones experimentales. 
la semejanza según las modalidades relevantes en cada fase de la investigación. Solamente en la prueba de transferencia extrarelacional se utilizó el criterio de igualación de diferencia. En la prueba de construcción se permitió que los participantes eligieran la relación a igualar. La tarea fue programada de tal manera que se controló el número de ensayos a igualar por cada relación.

\section{Diseño}

La tabla 1 sintetiza el diseño experimental que comprendió cinco fases: preprueba, entrenamiento, prueba de aprendizaje, pruebas de transferencia y prueba de construcción. Sólo los participantes con un desempeño menor al $50 \%$ de aciertos en la preprueba continuaron en el experimento. Estos participantes se asignaron aleatoriamente a uno de los tres grupos experimentales que diferían en el tipo de entrenamiento empleado: instrumental, instruccional u observacional. Sólo los participantes que lograron más del $87.5 \%$ de aciertos en la prueba de aprendizaje fueron expuestos a las pruebas de transferencia y de construcción. Con el propósito de alentar un alto desempeño en la prueba de aprendizaje, se les ofreció una recompensa económica a los participantes de Bogotá. En este caso, el investigador les informó que podrían recibir un pago, el cual dependería de su ejecución. Se les dijo que ganarían más si podían avanzar más en la tarea y, a su vez, que avanzarían más si podían resolverla de manera correcta. No obstante, al final de su participación el pago fue equivalente para todos los sujetos.

\section{Procedimiento}

Los participantes fueron recibidos por el investigador, quien les pidió que firmaran un consentimiento informado en el cual se describía de manera general la naturaleza del estudio, el tiempo que les tomaría participar y la voluntariedad de su participación.

\section{Fase 1. Preprueba}

Con el fin de garantizar la ingenuidad de los participantes en relación con la tarea, se incluyó esta fase como requisito para ser incluidos en el estudio. La preprueba contó con 24 ensayos de igualación sin retroalimentación. Los sujetos con desempeños inferiores al $50 \%$ pasaron a la siguiente fase, mientras que los que consiguieron más del $50 \%$ de aciertos fueron despedidos por el investigador, agradeciendo su participación. Al inicio de la preprueba apareció un texto en la pantalla en donde se informaba que el estudio realizado no evaluaba inteligencia, ni personalidad y únicamente investigaba procesos de aprendizaje comunes. Después aparecía en el monitor la siguiente instrucción acompañada de un botón con la palabra "Continuar":

En la pantalla aparecerán seis figuras: dos arriba, una en el centro y tres abajo. Debes elegir una de las figuras de abajo, haciendo clic sobre ella. La meta es lograr tantos aciertos como sea posible. Si tienes alguna pregunta hazla en este momento, pues más adelante no se te podrá suministrar información adicional.

\section{Fase 2. Entrenamiento}

Los participantes se expusieron a uno de los siguientes entrenamientos según el grupo al que fueron asignados.

Grupo 1: Exposición directa a la tarea. Se expuso a un entrenamiento compuesto por tres bloques de 24 ensayos cada uno. La mitad de los ensayos se estructuró de acuerdo con el criterio de igualación por semejanza, y la otra mitad por identidad. En cada ensayo se dio retroalimentación.

Tabla 1. Condiciones experimentales a las que se sometieron los participantes de los tres grupos.

\begin{tabular}{|c|c|c|c|c|c|}
\hline Grupo 1 & $\begin{array}{l}\mathrm{P} \\
\mathrm{R}\end{array}$ & $\begin{array}{l}\text { Entrenamiento con } \\
\text { exposición directa } \\
\text { a la tarea }\end{array}$ & & Pruebas de & \\
\hline Grupo 2 & $\begin{array}{l}\mathrm{C} \\
\mathrm{P} \\
\mathrm{R} \\
\mathrm{U} \\
\mathrm{E}\end{array}$ & $\begin{array}{l}\text { Entrenamiento con } \\
\text { observación de un } \\
\text { modelo aprendiz }\end{array}$ & $\begin{array}{c}\text { Prueba de } \\
\text { Aprendizaje }\end{array}$ & $\begin{array}{l}\text { - Intramodal } \\
\text { - Extramodal }\end{array}$ & $\begin{array}{l}\text { Prueba de } \\
\text { construcción }\end{array}$ \\
\hline Grupo 3 & $\begin{array}{l}\mathrm{B} \\
\mathrm{A}\end{array}$ & $\begin{array}{c}\text { Entrenamiento con } \\
\text { instrucciones } \\
\text { precisas }\end{array}$ & & - Extrarelacional & \\
\hline $\begin{array}{l}\text { Número de } \\
\text { sesiones }\end{array}$ & 1 & 3 & 1 & $\begin{array}{l}3 \text { (una por cada } \\
\text { tipo de prueba) }\end{array}$ & 1 \\
\hline $\begin{array}{l}\text { Número de } \\
\text { ensayos }\end{array}$ & 24 & 24 por sesión & 24 & 24 por sesión & 10 \\
\hline
\end{tabular}


Si la respuesta de igualación era la correcta, aparecía en la pantalla la palabra escrita "CORRECTO"; de lo contrario, aparecía "INCORRECTO". No se dio ningún tipo de instrucción adicional.

Grupo 2: Observación de un modelo aprendiz. Este grupo observó la ejecución de un modelo humano aprendiz que ejecutaba los mismos tres bloques de 24 ensayos con las mismas características del grupo 1. Sin embargo, a diferencia del grupo anterior, los participantes de este grupo únicamente observaron al modelo aprendiz. Este modelo fue un confederado que respondió con un patrón de respuesta tal que conseguía $41.6 \%$ de aciertos en la primera sesión, $70.8 \%$ de respuestas correctas en la segunda sesión y $91.7 \%$ de aciertos en la tercera sesión de entrenamiento. Los patrones de respuesta para las tres sesiones fueron diseñados de antemano por el investigador, distribuyendo los errores a lo largo del bloque de ensayos. En cada escenario experimental, el modelado fue responsabilidad de un único confederado, quien respondió con una latencia aproximada de cuatro segundos para cada ensayo con un movimiento de cursor regular. Con esto, se aseguró que los observadores fueran expuestos al mismo comportamiento de 'aprendizaje' por parte del confederado.

Grupo 3: Entrenamiento con instrucciones precisas. Los participantes de este grupo fueron expuestos a los mismos tres bloques de entrenamiento que se utilizaron en el grupo 1. Sin embargo, a diferencia del grupo 1, en cada ensayo se presentaron instrucciones precisas referidas a la relación de igualación vigente en dicho ensayo. Por ello, en un ensayo para igualar por semejanza en forma o color, se proporcionó la siguiente instrucción:

Teniendo en cuenta que las figuras de arriba son SEMEJANTES entre sí, escoge la figura de abajo que es SEMEJANTE a la figura del medio.

En un ensayo para igualar por identidad, el participante recibió la siguiente instrucción:

Teniendo en cuenta que las figuras de arriba son IDÉNTICAS entre sí, escoge la figura de abajo que es IDÉNTICA a la figura del medio.

Es importante señalar que los participantes asignados a este grupo no recibieron retroalimentación para poder evaluar el efecto de la sola instrucción sobre el aprendizaje de la discriminación condicional.

\section{Fase 3. Prueba de aprendizaje}

Consistió en 24 ensayos similares a los utilizados durante la fase de entrenamiento. Sin embargo, no se entregó retroalimentación a los participantes por sus elecciones ni se les proporcionaron instrucciones precisas sobre la relación a igualar. Considerando que lo que se transfiere es algo que se ha aprendido, se incluyó un criterio de exclusión para pasar a la fase siguiente de pruebas de transferencia. Es decir, sólo los participantes con porcentajes de aciertos iguales o mayores al $87.5 \%$ continuaban con pruebas de transferencia. Aquellos que no cumplieron con el criterio de desempeño fueron despedidos por el investigador, agradeciendo su participación.

Las instrucciones que recibieron todos los grupos fueron las siguientes:

Ahora desarrollarás una tarea similar a la anterior. Debes elegir una de las figuras de abajo haciendo clic sobre ésta. En esta ocasión NO se te dirá si tu elección es correcta o incorrecta, no obstante siempre habrá una respuesta correcta. Trata de acertar el mayor número de veces posible.

\section{Fase 4. Pruebas de transferencias}

Se emplearon tres tipos de pruebas de transferencia intramodal, extramodal y extrarelacional - cada una con 24 ensayos sin retroalimentación y sin ninguna instrucción que la antecediera. Así, los ensayos de pruebas de transferencia se presentaron sin nuevas instrucciones y sin señalar los cambios en las modalidades o criterios de igualación. Tampoco se proporcionó retroalimentación sobre sus aciertos al final de la prueba.

1. Prueba de transferencia intramodal. Se usaron los criterios de igualdad y semejanza pero se cambió la forma y el color de los estímulos comparativos y el estímulo muestra, manteniéndose en la misma modalidad (geométrica), pero con instancias diferentes a las usadas en el entrenamiento y en la prueba de aprendizaje (véase Figura 1).

2. Prueba de transferencia extramodal. Se presentaron como estímulos muestra y comparativos círculos de diferente tamaño y textura (véase Figura 1). Es decir, se conserva la dimensión geométrica pero se cambiaron las modalidades pertinentes a tomar en cuenta durante la igualación. Así, la relación de semejanza o identidad se estableció a partir del tamaño y la textura de las figuras geométricas.

3. Prueba de transferencia extrarelacional. Se usaron los mismos estímulos utilizados en el entrenamiento, pero en esta ocasión los estímulos de segundo orden prescribieron una relación de diferencia. Así, el estímulo comparativo correcto sería aquel que tuviera distinta forma y diferente color al estímulo muestra.

\section{Fase 5. Prueba de construcción}

Esta prueba estuvo conformada por diez ensayos en los cuales el participante debía armar un arreglo de igualación con los criterios que considerara pertinentes a partir de un número reducido de figuras geométricas. Tal como se describe en la figura 1, los estímulos de segundo orden fueron 
pentágonos y cruces de color café y rosa, mientras que los estímulos usados para los comparativos y el estímulo de muestra fueron estrellas que variaban en el número de puntas y el número de líneas internas (como efecto de la repetición de la forma de estrella al interior). Las opciones disponibles en cada ensayo le permitían realizar arreglos de igualación según diferentes criterios: identidad, diferencia, semejanza en forma, o semejanza en el número de repeticiones de la forma al interior.

Para armar los arreglos, se les presentó una pantalla inicial dividida en tres secciones (véase Figura 2, panel a). En la parte izquierda de la pantalla, apareció un arreglo de igualación incompleto. Es decir, sólo aparecía una cruz café en la posición del estímulo de segundo orden de la izquierda y recuadros para completar el resto de las posiciones de los estímulos. Esta sección de la pantalla inicial fue la misma para todos los ensayos y todos los participantes. Sin embargo, en la parte derecha de la pantalla aparecieron dos conjuntos de estímulos que variaron de ensayo a ensayo. El participante debía elegir el otro estímulo de segundo orden a partir del conjunto de cuatro figuras geométricas que aparecieron en la parte superior derecha. Además, debía acomodar un estímulo muestra y los tres estímulos comparativos de otro conjunto de cuatro figuras geométricas, las cuales se estructuraron al combinar estrellas de cuatro, cinco y seis puntas con texturas en donde se repetía al interior cero, una y dos veces la forma de estrella.

El participante debía completar el arreglo de igualación al arrastrar, mediante el ratón de la computadora, los estímulos disponibles en la sección derecha de la pantalla para acomodarlos dentro de los recuadros. Una vez armado el arreglo, el participante debía presionar el botón "Fin" que aparecía en el centro de la pantalla inicial. Enseguida, aparecían botones de selección debajo de cada estímulo comparativo (ver Figura 2, panel b). El participante debía señalar cuál de los estímulos de comparación era la respuesta correcta. Al hacerlo, pasaba al siguiente ensayo sin ningún tipo de retroalimentación. Al completar los diez ensayos, el programa le agradecía su participación y era despedido por el investigador (entregando el dinero en caso de que se le hubiera dicho que recibiría una compensación económica por su participación).

Al inicio y final de la prueba de construcción, se les pidió a los participantes que escribieran el criterio con el que se podía resolver la tarea correctamente. Esto con el propósito de recoger información adicional con respecto al conocimiento "declarativo" sobre la forma correcta de resolver la tarea. Los participantes escribieron sus estrategias de solución por medio del teclado y éstas se grabaron para su posterior análisis. Por ello, las instrucciones para esta fase se presentaron junto con una animación que mostraba la manera en que podía seleccionar, arrastrar y acomodar las figuras para armar el arreglo:

Teniendo en cuenta lo que acabas de escribir [verbalización antes de armar los arreglos], elabora unos ejemplos utilizando las figuras que encontrarás a continuación. Para elaborar tus ejemplos, selecciona las imágenes que se encuentran en el recuadro derecho y arrástralas con el cursor hasta los espacios que están disponibles en la parte izquierda. Posteriormente debes señalar cuál de las imágenes de abajo es la correcta con base en el arreglo que diseñaste. Observa el siguiente ejemplo.

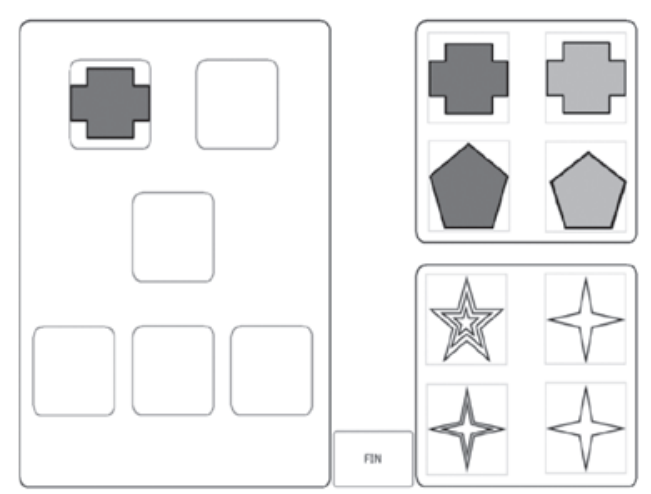

(a)
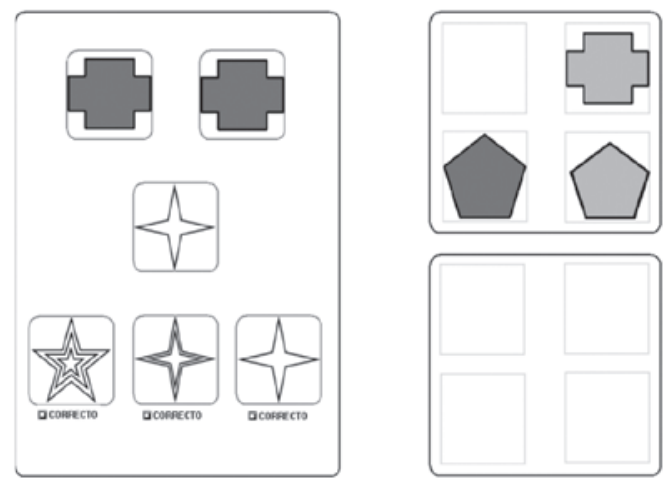

(b)

Figura 2. (a) Ejemplo de la pantalla inicial presentada al participante durante la fase de prueba de construcción. (b) Ejemplo de un posible arreglo que el participante pudiera armar durante la fase de prueba de construcción. En este caso, se trata de un arreglo a igualar por identidad y la respuesta correcta es la estrella de cuatro puntas sin repetición interna de la forma. 


\section{RESULTADOS}

Dado que el propósito del presente estudio fue evaluar el efecto de los diferentes tipos de entrenamiento sobre la adquisición, generalidad y transferencia de la discriminación condicional, los datos se analizaron con respecto al desempeño de los participantes, el número de ellos que logró responder a la prueba de aprendizaje con el criterio establecido, y su ejecución en las pruebas de transferencia, incluyendo la prueba de construcción. Las figuras 3 a 5 muestran el desempeño de cada uno de los participantes de los diferentes grupos. Las barras indican el porcentaje de aciertos de los participantes en las diferentes pruebas. Las líneas de puntos señaladas con E1, E2 y E3 indican porcentajes de acierto en los diferentes bloques de entrenamiento. No existen datos de entrenamiento para quienes únicamente observaron a un modelo aprendiz. Una línea punteada separa las ejecuciones de los participantes de los dos escenarios experimentales, Guadalajara y Bogotá. Dado que éstos últimos pudieron recibir un incentivo económico si lograban contestar con, al menos, $87.5 \%$ de aciertos en la prueba de aprendizaje, los grupos se distinguen en las gráficas de acuerdo con esta condición.

Los efectos del entrenamiento por exposición directa a la tarea se muestran en la figura 3. Sólo cinco de nueve participantes lograron cumplir el criterio de desempeño en la prueba de aprendizaje cuando no recibieron remuneración económica, mientras que sólo uno de los seis participantes que recibieron el incentivo económico logró superar el criterio de desempeño en la prueba de aprendizaje. Todos aquellos que pasaron a la fase de pruebas de transferencia lograron un porcentaje alto de aciertos en las pruebas.

Los efectos del entrenamiento por observación de un modelo aprendiz confederado se muestran en la figura 4 . Ninguno de los cinco participantes sin incentivo económico logró aprender la discriminación condicional. Sin embargo, cinco de siete que recibieron incentivo lograron aprenderla. Ellos tuvieron un buen desempeño en las pruebas de transferencia con excepción del participante P6 quien eligió el estímulo comparativo semejante (cuando la relación de igualación vigente era la diferencia) en la prueba de transferencia extrarelacional.

Todos los participantes, independientemente del escenario experimental, que recibieron instrucciones precisas lograron aprender la discriminación condicional (véase Figura 5). Sin embargo, algunos de ellos tuvieron un bajo desempeño en la prueba de transferencia extrarelacional. Por ejemplo, el participante $\mathrm{P} 8$ obtuvo $0 \%$ de aciertos ya que eligió exclusivamente la relación de semejanza a pesar de que los estímulos de segundo orden instruyeron una relación de diferencia.

Grupo 1: Entrenamiento con exposición directa
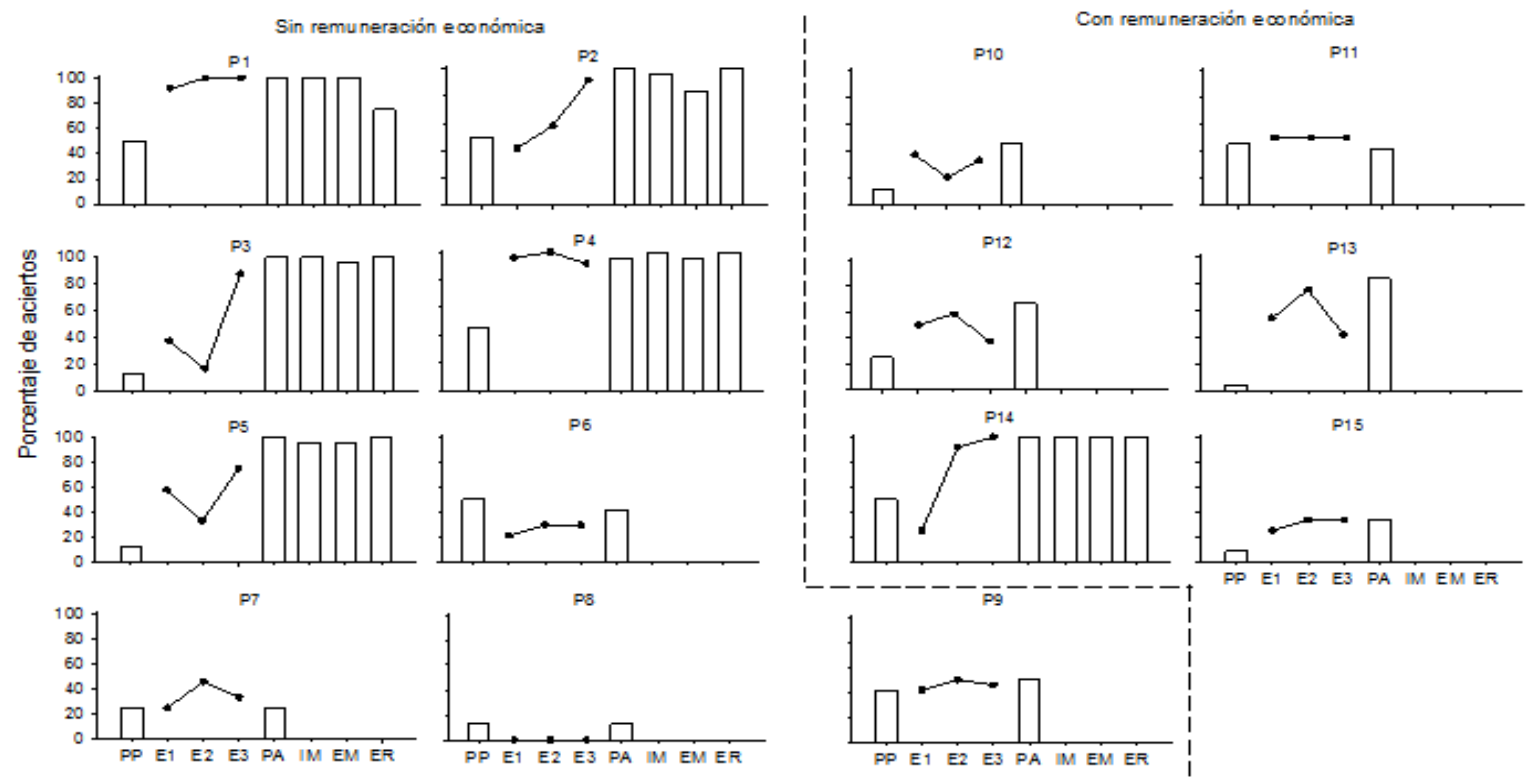

Figura 3. Porcentaje de aciertos de los participantes del grupo 1 (entrenamiento con exposición directa) sin remuneración económica (izquierda) y con remuneración económica (derecha). PP: Preprueba; E1-3: bloques de entrenamiento; PA: prueba de aprendizaje; IM: Prueba intramodal; EM: Prueba extramodal; ER: Prueba extrarelacional. 
Grupo 2: Entrenamiento con observación de modelo aprendiz
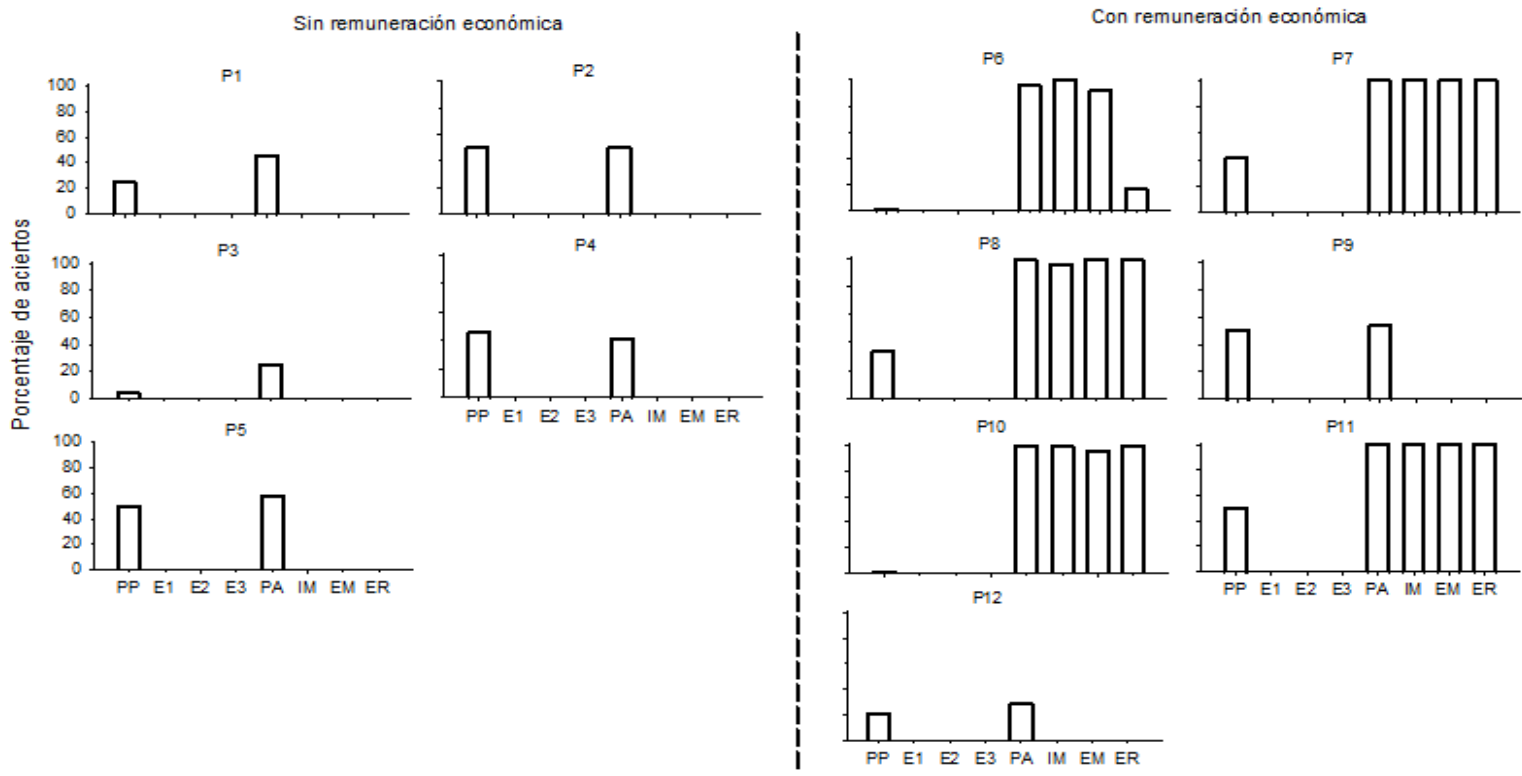

Figura 4. Porcentaje de aciertos de los participantes del grupo 2 (entrenamiento con observación del modelo aprendiz) sin remuneración económica (izquierda) y con remuneración económica (derecha). PP: Preprueba; E1-3: Bloques de entrenamiento; PA: Prueba de aprendizaje; IM: Prueba intramodal; EM: Prueba extramodal; ER: Prueba extrarelacional.

Grupo 3: Entrenamiento con instrucciones precisas
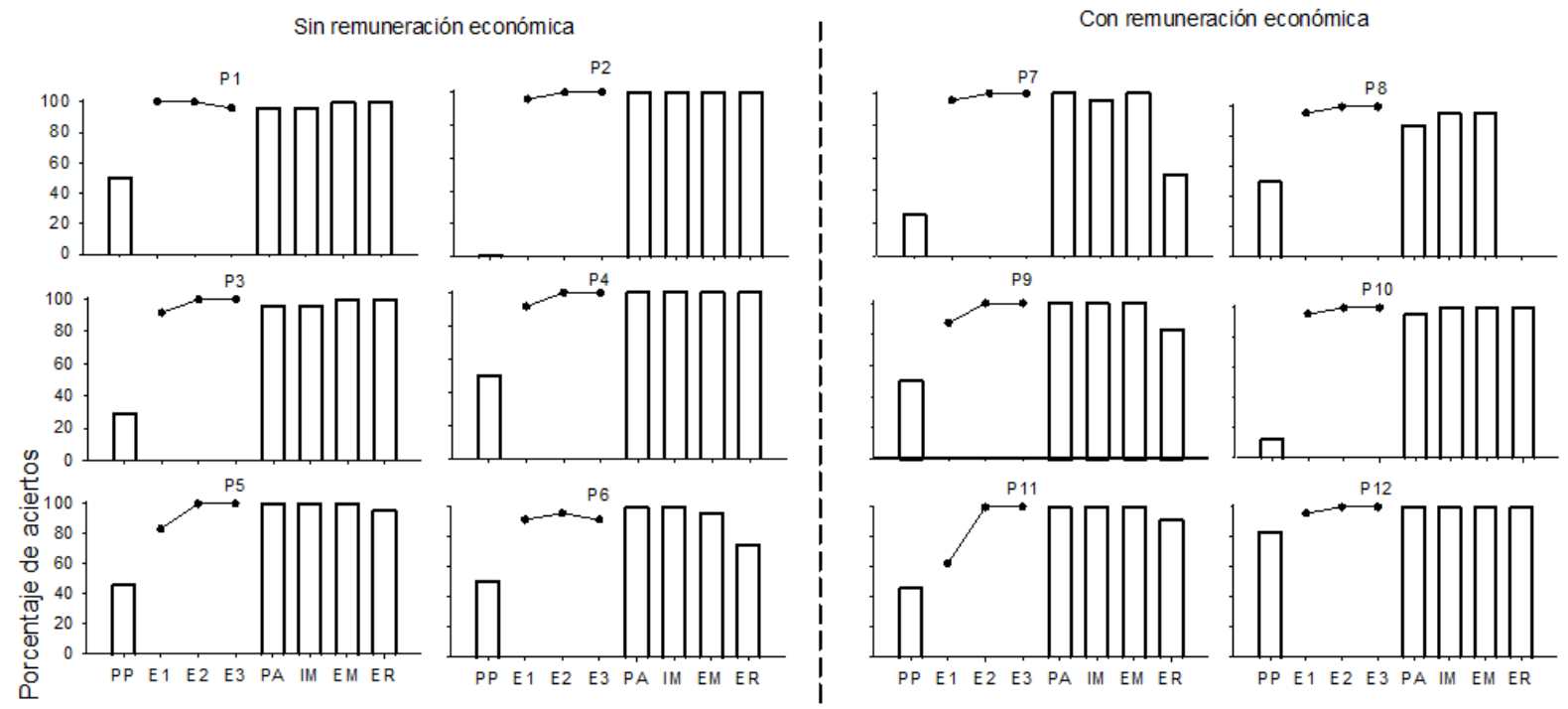

Figura 5. Porcentaje de aciertos de los participantes del grupo 3 (entrenamiento con instrucciones precisas) sin remuneración económica (izquierda) y con remuneración económica (derecha). PP: Preprueba; E1-3: Bloques de entrenamiento; PA: Prueba de aprendizaje; IM: Prueba intramodal; EM: Prueba extramodal; ER: Prueba extrarelacional. 
Al comparar el desempeño en las pruebas de transferencia de quienes lograron resolver la prueba de aprendizaje con el criterio establecido, se puede observar que todos respondieron a las pruebas de transferencia intramodal y extramodal con más de $95 \%$ de aciertos. Sin embargo, el grupo entrenado con exposición directa a la tarea consiguió en promedio $96 \%$ de aciertos (desviación estándar $=10$ ) en la prueba extrarelacional, mientras que el grupo que observó sólo logró $82 \%$ de aciertos (desviación estándar = 37) y el grupo que recibió instrucciones precisas tuvo en promedio $83 \%$ de aciertos (desviación estándar $=30$ ). Es decir, aunque los participantes que recibieron instrucciones precisas aprendieron más rápido, quienes se expusieron a la tarea directamente lograron un mejor desempeño en la prueba de transferencia extrarelacional.

Con el propósito de comparar los desempeños de los participantes en la prueba de aprendizaje, se calculó cuántos de ellos aprendieron la discriminación condicional en cada grupo. Se encontró que el $56 \%$ de los participantes del grupo entrenado mediante exposición directa sin incentivo logró ese aprendizaje, mientras que esto sólo ocurrió en el $17 \%$ de su contraparte que recibió incentivo. Para la condición de observación del modelo aprendiz, ningún participante del grupo sin incentivo logró aprender la discriminación, mientras que el $71 \%$ de quienes recibieron incentivo sí lo logró. Todos los participantes aprendieron cuando recibieron instrucciones precisas sin importar si se les hubiera dado o no, un incentivo. Se llevó a cabo una prueba de chi cuadrada con estos porcentajes y se encontraron diferencias estadísticamente significativas $\left(\chi^{2}=89.6\right.$, g.l. $\left.=2, p<0.0001\right)$. Es decir, la entrega de la remuneración económica tuvo un efecto diferencial sobre la prueba de aprendizaje según el tipo de entrenamiento utilizado. No influyó de manera positiva cuando se emplearon los entrenamientos por exposición directa a la tarea o a las instrucciones precisas, pero sí tuvo un efecto positivo en el entrenamiento mediante la observación de un modelo aprendiz.

Por último, se llevó a cabo un análisis de la ejecución de los participantes en la prueba de construcción y de sus verbalizaciones antes y después de dicha prueba. Para ello, en cada ensayo armado se identificó: (1) la relación de igualación mostrada por los estímulos de segundo orden y (2) la relación de igualación entre el estímulo muestra colocado y el estímulo comparativo que el participante señaló como correcto. Un arreglo se consideraba válido si la relación en (1) era la misma relación que en (2). La tabla 2 describe el tipo de ensayos que armaron los participantes cuando lograron arreglos válidos. Como se aprecia en dicha tabla, casi la mitad de los ensayos construidos mostraron la relación de identidad. Además, casi todos los participantes armaron los diez ensayos con arreglos de igualación válidos. En el grupo donde hubo más ensayos con arreglos no válidos fue el que se entrenó mediante el uso de instrucciones precisas. Con respecto a las descripciones de los participantes antes y después del armado de ensayos de igualación, es importante señalar que éstas no constituyeron un indicador del desempeño terminal de los participantes ni de su ejecución en la prueba de construcción.

\section{DISCUSIÓN}

La propuesta de Ribes y López (1985) sugiere que es posible reorganizar diversos fenómenos, datos o registros producidos en el contexto de la investigación empírica en múltiples áreas de la psicología en virtud de sus propiedades funcionales. En ese contexto, Ribes (1990) afirma que es posible encontrar equivalencias funcionales entre diversos métodos o procedimientos para el desarrollo de competencias, entendidas como tendencias al desempeño efectivo y variado, que en el ámbito educativo formal suelen asumir usualmente la forma de enseñanza por demostración, por exposición directa con respecto a aspectos particulares de la situación de enseñanza (que no a las contingencias, dado que en todos los casos habría exposición directa a las contingencias) y por instrucción.

Por ello, en el presente estudio se comparó la efectividad de tres entrenamientos distintos para propiciar el aprendizaje de una discriminación condicional mediante una tarea de igualación a la muestra de segundo orden. Un grupo se entrenó mediante la exposición directa a la tarea. Es decir, recibió instrucciones mínimas pero su ejecución se retroalimentó en cada ensayo de entrenamiento. Otro grupo tuvo que observar la ejecución de un modelo (confederado) mientras 'aprendía' la discriminación condicional. Por último, un tercer grupo recibió textos en cada ensayo con la instrucción precisa de cuál relación de igualación estaba vigente. Para estimar el efecto aislado de la instrucción, este grupo no recibió retroalimentación de su ejecución.

Algunos de los hallazgos encontrados en esta investigación son consistentes con estudios previos que comparan los efectos de la retroalimentación y las instrucciones en tareas de discriminación condicional. Por ejemplo, el empleo de instrucciones precisas tuvo como resultado que los participantes aprendieran la discriminación muy rápidamente, pero, algunos de ellos cometieron errores en la prueba de transferencia extrarelacional y en la prueba de construcción de arreglos de igualación válidos. Así, los que aprendieron bajo la exposición directa a la tarea lograron un mejor desempeño en la transferencia extrarelacional que los que aprendieron mediante la observación del modelo aprendiz o las instrucciones precisas. Es decir, un "ajuste" 
Tabla 2.

Desempeño de los participantes que se expusieron a la fase de prueba de construcción en la cual debían armar arreglos de igualación de la muestra de segundo orden válidos a partir de los estímulos proporcionados por el experimentador.

\begin{tabular}{|c|c|c|c|c|c|c|}
\hline \multirow{2}{*}{ Grupo } & \multirow{2}{*}{ Participante } & \multicolumn{4}{|c|}{$\begin{array}{c}\text { Número de arreglos válidos según la relación } \\
\text { de igualación empleada }\end{array}$} & \multirow{2}{*}{$\begin{array}{l}\text { Total de } \\
\text { arreglos válidos } \\
\text { por participante }\end{array}$} \\
\hline & & Identidad & $\begin{array}{l}\text { Semejanza en } \\
\text { Forma }\end{array}$ & $\begin{array}{l}\text { Semejanza en } \\
\text { Color/Textura }\end{array}$ & Diferencia & \\
\hline \multirow{6}{*}{$\begin{array}{l}\text { Exposición } \\
\text { directa }\end{array}$} & P1 & 2 & 4 & 2 & 2 & 10 \\
\hline & P2 & 3 & 2 & 2 & 3 & 10 \\
\hline & P3 & 7 & 1 & 0 & 2 & 10 \\
\hline & P4 & 4 & 3 & 1 & 2 & 10 \\
\hline & P5 & 3 & 3 & 1 & 3 & 10 \\
\hline & P14 & 4 & 2 & 2 & 2 & 10 \\
\hline \multirow{5}{*}{ Modelado } & P6 & 8 & 0 & 1 & 1 & 10 \\
\hline & P7 & 3 & 4 & 0 & 3 & 10 \\
\hline & P8 & 4 & 2 & 2 & 2 & 10 \\
\hline & P10 & 3 & 2 & 2 & 3 & 10 \\
\hline & P11 & 4 & 1 & 1 & 3 & 9 \\
\hline \multirow{12}{*}{$\begin{array}{c}\text { Instrucciones } \\
\text { precisas }\end{array}$} & P1 & 5 & 3 & 0 & 2 & 10 \\
\hline & $\mathrm{P} 2$ & 3 & 3 & 2 & 1 & 9 \\
\hline & P3 & 6 & 2 & 0 & 1 & 9 \\
\hline & P4 & 2 & 4 & 2 & 2 & 10 \\
\hline & P5 & 3 & 1 & 3 & 3 & 10 \\
\hline & P6 & 6 & 2 & 0 & 1 & 9 \\
\hline & P7 & 3 & 1 & 4 & 1 & 9 \\
\hline & P8 & 7 & 0 & 2 & 0 & 9 \\
\hline & P9 & 9 & 1 & 0 & 0 & 10 \\
\hline & P10 & 3 & 4 & 3 & 0 & 10 \\
\hline & P11 & 7 & 0 & 2 & 0 & 9 \\
\hline & $\mathrm{P} 12$ & 5 & 0 & 1 & 1 & 7 \\
\hline \multicolumn{2}{|c|}{$\begin{array}{l}\text { Total de arreglos válidos por cada } \\
\text { relación de igualación }\end{array}$} & 104 & 45 & 33 & 38 & 220 \\
\hline
\end{tabular}

más rápido y efectivo a la situación no supone control abstracto del estímulo ni la transferencia de lo aprendido a nuevos eventos conductuales (Ribes, 2000).

Los efectos de los tipos de entrenamiento fueron distintos en los dos escenarios experimentales sin que ninguna población de participantes fuera mejor que la otra. Esta diferencia en los resultados podría ser un derivado de las distintas historias académicas de los participantes de cada grupo. Sin embargo, por los datos reportados en la presente investigación y en el estudio de Vega y Peña (2008), tal parece que la procedencia académica de los participantes no es una variable relevante. Es decir, los participantes en Vega y Peña venían de un contexto académico similar a los que aquí se emplearon en el escenario experimental de Bogotá. Aún así, un alto porcentaje de los participantes del presente estudio logró aprender la discriminación en la condición de observación de un modelo aprendiz mientras que el empleo de modelos aprendices experimentales en Vega y Peña (2008) no favoreció el aprendizaje de la discriminación condicional. Esto lleva a considerar que las diferencias en el aprendizaje de los participantes de las dos poblaciones aquí estudiadas puedan deberse a la entrega de la remuneración económica. Así, es deseable replicar la condición de entrenamiento observacional, con y sin incentivo, en participantes de un mismo contexto académico. De esta manera, también se podrían controlar otras posibles variables extrañas vinculadas al modelo confederado, a la frecuencia y distribución de las instancias de retroalimentación positiva y negativa en cada condición, y al valor de la recompensa recibida.

Se puede decir, con respecto a la investigación sobre el efecto del modelamiento de la ejecución, que ésta ha estado centrada en la reproducción de rutinas conductuales (Neef, Marckel, Ferreri, Jung, Nist \& Armstrong, 2004) o como forma de enseñanza para promover el control del aprendizaje social como en los casos de imitación o facilitación social (Bandura, Grusec \&Menlove, 1966; Fisher \& Kodak, 2007;Geshuri,1975). Esto dificulta la comparación de tales 
estudios con la presente investigación, dado que el aprendizaje rutinario no implica la identificación de relaciones entre estímulos distintos a la conducta del modelo y, por tanto, no implica el control abstracto de estímulo. Sin embargo, hay dos cuestiones de interés teórico que pueden resaltarse de dichos estudios. La primera es la postura explícita de que el aprendizaje se puede ver afectado por variables de tipo motivacional como la entrega de reforzadores o incentivos. La segunda cuestión tiene que ver con una noción de que un procedimiento de modelamiento es más efectivo si se acompaña de descripciones de la propia conducta por parte del modelo. Por tanto, en futuras investigaciones se podrían incorporar estas consideraciones teóricas al tiempo que se emplea una tarea que exige el control abstracto de estímulo.

Es importante señalar que la variable de la remuneración económica surgió coyunturalmente en la presente investigación. Sin embargo, los resultados sugieren que el empleo de incentivos puede operar en interacción con las variables relevantes en el aprendizaje de una discriminación condicional. Es decir, la recompensa económica puede funcionar como un factor motivacional o disposicional (dado que afecta la probabilidad del establecimiento de control abstracto en estas condiciones), especialmente en las condiciones de mayor dificultad. No obstante, también es posible afirmar que el papel del incentivo puede ser modulado por la dificultad del requisito conductual de una tarea particular. En cualquier caso, este factor parece tener efectos dignos de estudiarse, o cuando menos de controlarse en investigaciones futuras.

Sin embargo, en términos generales con respecto a la pregunta de investigación que guió originalmente el presente trabajo, es posible afirmar que, a pesar de la variabilidad interindividual, el logro conductual de control abstracto resulta más difícil en condiciones de aprendizaje por observación (en ausencia de incentivo), lo que parece no ser consistente con estudios como los de Tonneau y cols. (2006). No obstante, esta diferencia puede ser atribuida a las particularidades del procedimiento (número de ensayos, número de bloques, requisito de respuesta, incentivo, etc.).

En comparación con las otras condiciones, es posible afirmar que la situación de modelamiento, si bien no difiere en el número de componentes contingenciales implicados, sí puede sugerir un papel diferencial de la respuesta de igualación observada en comparación con el papel de la respuesta directa o de la instrucción. En otras palabras, es posible que el aprendizaje observacional de relaciones entre estímulos, al menos en ausencia de incentivos, implique funciones conductuales diferentes, y en ese contexto, la elaboración de reglas verbales sea una condición que permita o promueva el "contacto indirecto" con aquello que es directo en las otras dos condiciones, lo que sugiere otra vía de indagación relevante.
Además, es importante discutir sobre el empleo de una prueba de construcción como la descrita en la presente investigación para evaluar el dominio que tiene un individuo sobre la 'regla' en operación en una tarea de igualación de la muestra. En los primeros estudios del área, se esperaba que las descripciones del desempeño efectivo correspondieran con el desempeño en las pruebas de transferencia. Es decir, uno de los supuestos con los que se ha trabajado empíricamente es que las pruebas de transferencia (especialmente las que suponen mayor complejidad, como la prueba de transferencia extrarelacional y extradimensional) permiten evaluar un comportamiento mediado lingüísticamente (Hurtado, Robayo \& Peña, 2007). En este sentido, la falta de correspondencia entre los niveles de desempeño y la precisión de las descripciones ha hecho que se cuestione el papel 'posibilitador' de las descripciones en la estructuración del comportamiento complejo (González \& Ortiz, 2014). En la presente investigación, no sólo se registraron las verbalizaciones que hicieron los participantes sino que se les pidió que armaran arreglos válidos de igualación a la muestra. De acuerdo con tales verbalizaciones, y tal como sugieren los resultados, esta construcción sí correlacionó con la ejecución en la prueba de transferencia extrarelacional. En contraste, el análisis de las verbalizaciones no arrojó ningún hallazgo a reportar. Dado que el desempeño en este procedimiento de armar arreglos válidos de igualación a la muestra parece estar mejor correlacionado con la ejecución en pruebas de transferencia, considerando su nivel de complejidad supuesto, dicha metodología puede ser una opción experimental viable en el estudio de la discriminación condicional mediada lingüísticamente.

Por último, hay que hacer una acotación sobre la naturaleza conceptual de la prueba de construcción. Bautista (2010) originalmente implementó un procedimiento de dos ensayos en donde se emplearon números para armar ensayos de igualación. Para cada posición en el arreglo, se proporcionaron tres opciones y el participante debía elegir cuál estímulo (de los tres disponibles) debía ir en cada espacio. En este sentido, se trató de una prueba de construcción "extra instancia y extra dimensional". Sin embargo, en el presente estudio, para simplificar el procedimiento y poder incluir diez ensayos de construcción de arreglos, se emplearon figuras geométricas distintas (y no números). Así, podría decirse que se empleó una prueba de construcción "extra instancia y extra modal". En ambos casos, la ejecución adecuada en la prueba de construcción puede ser interpretada como una muestra de ampliación funcional, ya que implica una transferencia a una situación novedosa en donde se interactúa legítimamente en función de la enunciación verbal (Bautista, 2010). Esa es otra razón para incorporar pruebas de transferencia de construcción 
de relaciones en el estudio del efecto de variables lingüísticas en la estructuración del comportamiento humano.

\section{REFERENCIAS}

Bandura, A., Grusec, J. E., \& Menlove, F. L. (1966). Observational learning as a function of symbolization and incentive set. Child Development, 37, 499-506.

Bautista, R. (2010). Efectos de diversos aspectos de la retroalimentación asociada con la producción de instrucciones en tareas de discriminación condicional de segundo orden. Tesis de Maestría. Universidad Nacional de Colombia.

Fisher, W. W., \& Kodak, T. (2007). Embedding an identitymatching task within a prompting hierarchy to facilitate acquisition of conditional discriminations in children with autism. Journal of Applied Behavior Analysis, 40, 489-499.

Geshuri, Y.(1975). Discriminative observational learning: Effects of observed reward and dependency. Child Development, 46, 550-554.

Goldiamond, I. (1966). Perception, language, and conceptualization rules. En B. Kleinmuntz (Coord.), Problem solving: Research, methods and theory (pp. 183-224). Nueva York: Wiley.

González, V., \& Ortiz, G. (2014). Efectos del tipo y contenido de las descripciones precontacto sobre la conducta de discriminación condicional y las descripciones postcontacto. Acta Colombiana de Psicología, 17(1), 11-23. Doi:10.14718/ACP.2014.17.1.2.

Guzmán-Díaz, G., \& Serrano, M. (2013). Propiedades fisicoquímicas versus lingüísticas en la adquisición y transferencia de discriminaciones condicionales por humanos. Acta Comportamentalia, 21(4), 435-444.

Hurtado, C., Robayo, M. A. \& Peña, T. E. (2007). Efectos en la ejecución durante una tarea de igualación a la muestra según el tipo y el orden de exposición a las pruebas de transferencia. Universitas Psychologica, 6(2), 425-440.

Islas, A., \& Flores, C. (2007). Papel de la retroalimentación en la adquisición y transferencia de discriminaciones condicionales en estudiantes universitarios. Enseñanza e Investigación en Psicología, 12(1), 65-77.

Neef, N., Marckel, J., Ferreri, S., Jung, S., Nist, L. \& Armstrong, N. (2004). Effects of modeling versus instructions on sensitivity to reinforcement schedules. Journal of Applied Behavior Analysis, 37(3), 267-281.

Ortiz, G., \& González, V. (2010). Efecto de dos tipos de descripciones precontacto sobre la ejecución instrumental y descripciones poscontacto en tareas de igualación de la muestra. Acta Colombiana de Psicología, 13(1), 115-126.

Ortiz, G., González, A., Rosas, M., \& Alcaráz, F. (2006). Efectos de la precisión instruccional y la densidad de retroalimentación sobre el seguimiento, la elaboración y transmisión de descripciones en tareas de discriminación condicional. Acta Comportamentalia, 14(2), 103-130.
Pérez, R., \& Pereira, F. (1986). Conducta gobernada por la regla y ejecución en un proceso de discriminación I. Aproximación al problema. Revista Latinoamericana de Psicología, 18(1), 57-71.

Ribes, E. (1990). Psicología General. México: Trillas.

Ribes, E. (2000). Instructions, rules and abstraction: A misconstrued relation. Behavior and Philosophy, 28, 41-55.

Ribes, E., \& Castillo, A. (1998). Interacción del tipo de entrenaiento y el tipo de respuesta de igualación en transferencia en una discriminación condicional de segundo orden. Acta Comportamentalia, 6, 5-20.

Ribes, E., Ibáñez, C. \& Hernández-Pozo, R. (1986). Hacia una psicología comparativa: Algunas consideraciones conceptuales y metodológicas. Revista Latinoamericana de Psicología, 18, 263-276.

Ribes, E., \& López, F. (1985). Teoría de la conducta: Un análisis de campo y paramétrico. México: Trillas.

Ribes, E., \& Martínez, H. (1990). Interaction of contingencies and rule instructions in the performance of human subjects in conditional discrimination. The Psychological Record, 40, 565-586.

Ribes, E., Moreno, D., \& Martínez, C. (1998). Second-order Discrimination in humans: the roles of explicit instructions and constructed verbal responding. Behavioural Processes, $42,1-18$.

Ribes, E., Rodríguez, M. E. \& Fuentes, T. (2003). Anticipating the correct matching response in a second-order matchingto-sample task. Psychological Reports, 93, 1307-1318.

Ribes, E., Torres, C., Barrera, J., \& Ramírez, L. (1995). Efectos de la variación modal de los estímulos en la adquisición y transferencia de una discriminación condicional en humanos adultos. Acta Comportamentalia, 3(2), 115-151.

Ribes, E. \& Zaragoza, A. (2009). Efectos de las instrucciones y descripciones con sin criterios en la adquisición y transferencia de una discriminación condicional de segundo orden. Acta Comportamentalia, 17(1), 61-95.

Skinner, B. (1979). Contingencias de reforzamiento (E. Galindo Cota, trad.). México: Trillas. (Obra original publicada en 1969)

Tonneau, F.; Arreola, F.; \& Martínez, A.G. (2006). Function transformation without reinforcement. Journal of the Experimental Analysis of Behavior, 85(3), 393-405.

Tonneau, F., \& González, C. (2004). Function transfer in human operant experiments: The role of stimulus pairings. Journal of the Experimental Analysis of Behavior, 81(3), 239-255.

Varela, J. \& Quintana, C. (1995). Comportamiento inteligente y su transferencia. Revista Mexicana de Análisis de la Conducta, 21, 47-66.

Vega, M., \& Peña, T. (2008). Efecto de diferentes entrenamientos sobre el aprendizaje, transferencia y formulación de la regla en una tarea de igualación a la muestra de primer orden. Revista Colombiana de Psicología, 17(2), 115-127.

Villanueva, S., Mateos, R., \& Flores, C. (2008). Efectos del contenido y distribución de la retroalimentación sobre la discriminación condicional de segundo orden. Acta Comportamentalia, 16(2), 211-221. 